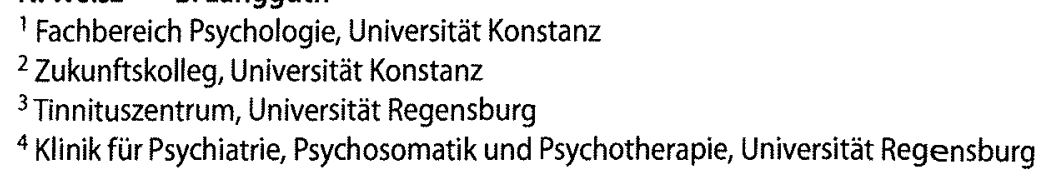

\title{
Kortikale Plastizität und Veränderungen bei Tinnitus
}

\author{
Therapeutische Optionen
}

\begin{abstract}
Tinnitus schneidet eine der spannendsten Fragen der Neurowissenschaften an: Wie entsteht eine bewusste und oft leidvolle Wahrnehmung allein aus intrinsischer Hirnaktivität, d. h. unabhängig von extern messbaren Signalen? Aus klinischer Sicht entscheidender ist jedoch die Frage, ob diese abnormale Hirnaktivität - und somit die Wahrnehmung des Geräuschs - veränderbar bzw. gar rückgängig zu machen ist? Beide Fragen sollen in unserem Beitrag angeschnitten werden. Wo steht die aktuelle Hirnforschung zu Tinnitus und welche therapeutischen Optionen ergeben sich daraus?
\end{abstract}

\section{Entstehung im Gehirn}

Subjektiver Tinnitus erfüllt alle Kriterien einer bewussten akustischen Wahrnehmung (u. a. Lokalisation, Intensität und Spektrum), allerdings ist keine externe oder körpereigene Schallquelle identifizierbar. Unabhängig davon liegen jedoch genauso wie jeder anderen bewussten Wahrnehmung neurophysiologische Korrelate zugrunde. Ursprüngliche Annahmen, dass Tinnitus bedingt ist durch eine erhöhte Spontanaktivität des Hörnervs, konnten experimentell nicht bestätigt werden [1].

Entsprechend wird in aktuellen neurowissenschaftlichen Erklärungsmodellen angenommen, dass nicht ein gestei- gertes Signal aus der Peripherie Tinnitus verursacht, sondern dass eher verminderte Information aus dem Innenohr entscheidend ist für seine Entstehung. Hörverlust ist der wichtigste Risikofaktor für die Entstehung von Tinnitus, und bei etwa $60 \%$ der Betroffenen lassen sich mit Standardaudiometrien messbare EinbuBen in der Hörfähigkeit feststellen. Sogar bei Betroffenen mit wenig oder gar keinem subjektiven Hörverlust kann man mit sensitiveren Verfahren häufig Defizite nachweisen [2]. Des Weiteren erscheint nicht die absolute Ausprägung des Hörverlusts ausschlaggebend zu sein, sondern die Stärke von Diskontinuitäten (Steilheit der Audiogrammkurve) in der Hörfähigkeit [3].

Auf der Basilarmembran benachbarte Haarzellen kodieren für benachbarte Frequenzen. Diese sog. tonotope Organisation setzt sich in der gesamten zentralen Hörbahn fort, sodass im auditorischen Kortex benachbarte Frequenzen ebenfalls nebeneinander repräsentiert sind. Die Ausbreitung von neuronaler Erregung wird in der zentralen Hörbahn über hemmende und erregende modulierende Systeme kontrolliert. $\mathrm{Zu}$ den wichtigsten hemmenden Systemen gehört das System der lateralen Inhibition: Die Aktivierung einer bestimmten Frequenz führt zur Hemmung der benachbarten Frequenzen. Starke Diskontinuitäten im Hörvermögen führen $\mathrm{zu}$ einer gestörten exzitatorisch-inhibitorischen Balance zwischen benachbarten normal afferent innervierten und deprivierten Neuronen (a Abb. 1). So wurden unmittelbar nach einem Knalltrauma Veränderungen der neuronalen Aktivität im auditorischen Kortex nachgewiesen, wobei sich zunächst gesteigerte Synchronisation neuronaler Aktivität erhöht und erst später die Feuerungsrate [4]

Aus humanexperimenteller Sicht ist dieser Befund von großer praktischer Bedeutung: Noninvasive Methoden wie Magneto- und Elektroenzephalographie (MEG bzw. EEG) können lediglich die synchronisierte Aktivierung größerer Neuronenverbünde messen. Somit erscheint die Untersuchung neuronaler Korrelate von Tinnitus beim Menschen mit EEG oder MEG prinzipiell möglich. Synchronisierte Aktivität weist meist kein unkoordiniertes, sondern ein rhythmisches Muster auf. Daher erscheint die Untersuchung sog. oszillatorischer Aktivität besonders vielversprechend.

\section{O Synchronisierte \\ Aktivität weist meist ein rhythmisches Muster auf}

MEG-Untersuchungen der Konstanzer Arbeitsgruppe (s. [5] für einen Überblick) und EEG-Untersuchungen anderer Arbeitsgruppen $[6,7]$ zeigen bei chronischen Tinnitusbetroffenen im Ruhezustand eine Reduktion des normalerweise dominanten $\alpha$-Rhythmus (8-12 Hz) über temporalen Regionen, während lang- 


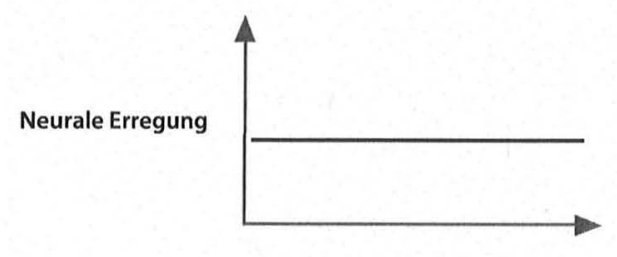

Tonotopie
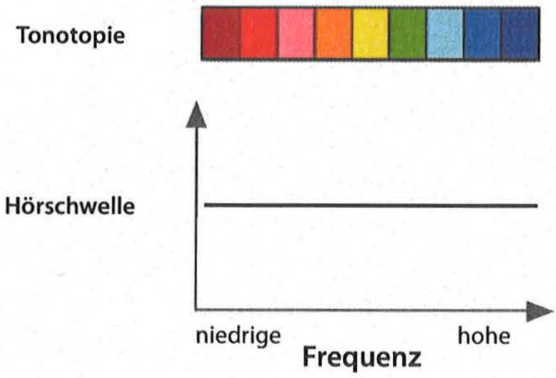

\section{Tinnitus und Hörminderung}
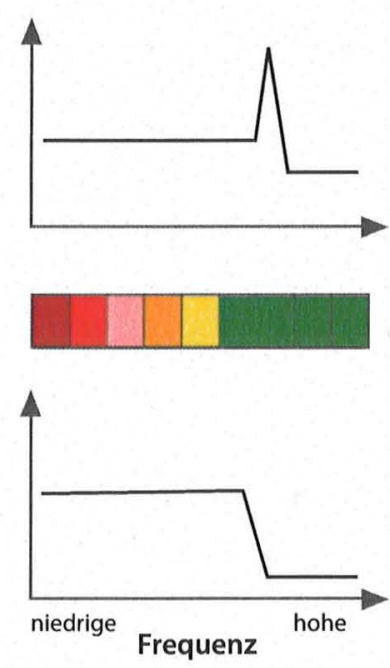

Abb. $1 \Delta$ Schematische Darstellung veränderter Hörfähigkeit, Tonotopie sowie Spontanaktivität von Neuronen im auditorischen Kortex

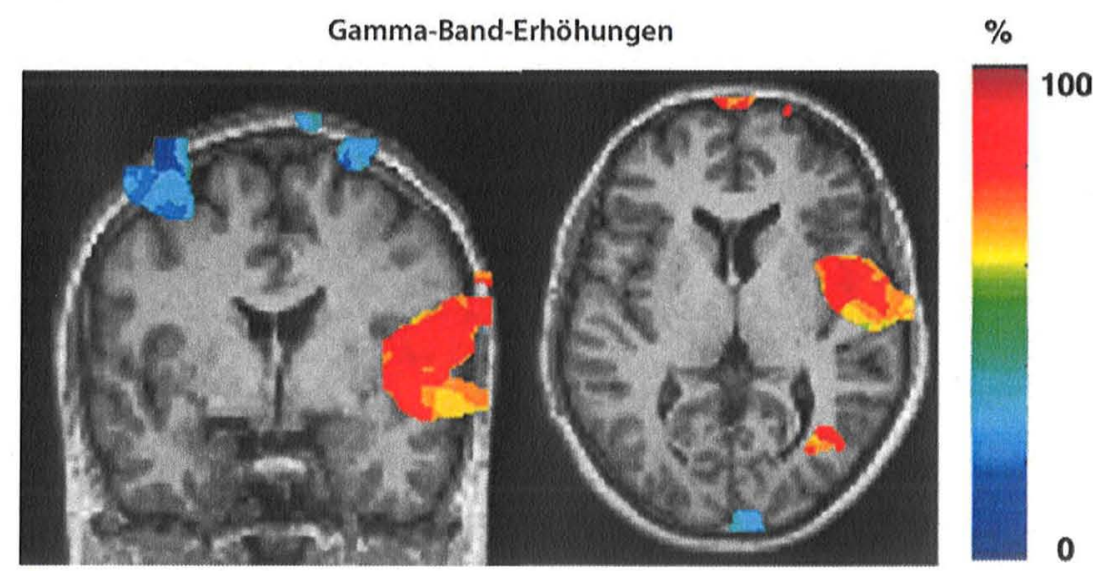

Abb. $2 \Delta$ Anstieg der y-Aktivität im rechten auditorischen Kortex nach Lärm

samere $[\delta-($ Delta-) $/ \theta$-(Theta-)Rhythmen; $1-6 \mathrm{~Hz}]$ und schnelle [ $\gamma$-(Gamma-) Rhythmus; >30 Hz] Rhythmen deutlich erhöht sind (s. auch thalamokortikale Dysrhythmien; [8]). Die $\delta$ - $/ \theta$-Rhythmen im auditorischen Kortex bei Tinnitusbetroffenen könnten demnach das Korrelat der akustischen Deprivation durch den Hörverlust darstellen. $\alpha$-Aktivität hingegen scheint Ausdruck funktioneller Inhibition zu sein. So geht starke a-Aktivitäl vor der Präsentation eines Reizes oder eines TMS-Pulses u. a. mit schlechteren Detektionsleistungen [9] bzw. kleineren motorisch evozierten Potenzialen [10] einher. Bei gesunden Probanden ist in Ruhe der
a-Rhythmus die dominierende Aktivität in sensorischen und motorischen Regionen, die sich nach sensorischer Stimulation bzw. einem motorischen Akt reduziert (sog. „Desynchronisation“).

Wir haben früher [5] die Hypothese formuliert, dass das dominante Ruhe- $\alpha$ das Produkt von Mechanismen ist, die eine „spontane Synchronisierung" von exzitatorischen Neuronen unterdrücken. Die bei Tinnituspatienten nachgewiesene verminderte $\alpha$-Aktivität in auditorischen Regionen könnte so ein durch den Hörverlust verursachtes inhibitorisches Defizit widerspiegeln. Die bisher beschriebenen Mechanismen - so wichtig sie möglicher- weise für Tinnitus sind - stellen jedoch noch nicht das neuronale Korrelat der Phantomwahrnehmung selbst dar.

Als Mindestanforderung für das neuronale Korrelat von Tinnitus gilt, dass - die Aktivierung kontinuierlich vorhanden sein müsste und

- mit einer erhöhten Erregung zusammenhängt, die von höhergeordneten wahrnehmungsrelevanten Hirnregionen verarbeitet wird.

In diesem Zusammenhang ist die Beobachtung erhöhter $\gamma$-Aktivität bei Tinnituspatienten besonders interessant. Aus tierexperimentellen Arbeiten ist bekannt, dass $\gamma$-Oszillationen stark mit schneller synchroner Feuerung von Neuronen zusammenhängen [11]. Konzeptuell ist diese Form der Aktivität für Tinnitus relevant. Zum einen wird vermutet, dass $\gamma$ Oszillationen die Aktivität von verteilten Neuronen(gruppen) synchronisieren und somit die von ihnen verarbeiteten Merkmale zu einer kohärenten Repräsentation „binden“ [12]. Des Weiteren hat die schnelle synchronisierte Aktivierung einer Neuronengruppe einen größeren postsynaptischen Einfluss als eine unsynchronisierte Aktivierung. Im Kontext der Tinnituserkrankung haben $\gamma$-synchronisierte Neurone im auditorischen Kortex eine erhöhte Wahrscheinlichkeit, Hirnregionen zu beeinflussen, die z. B. für Aufmerksamkeit bzw. bewusste Wahrnehmung relevant sind. Zahlreiche Befunde belegen, dass bewusst im Gegensatz zu nicht bewusst wahrgenommenen Reizen [13] mit crhöhter $\gamma$-Aktivität in sensorischen Hirnregionen einhergehen. Aufgrund dieser Befunde könnte die Hypothese aufgestellt werden, dass $\gamma$-Oszillationen im auditorischen Kortex das grundlegende neuronale Korrelat von Tinnitus darstellen. Auch wenn diese Hypothese plausibel erscheint, ist die experimentelle Evidenz dafür bisher schwach. Vor allem gelang es bisher nicht, einen eindeutigen Zusammenhang zwischen der Lateralität der Wahrnehmung (s. [14] für eine Ausnahme bei durch langsame Wellen modulierten $\gamma$-Oszillationen) und der Lateralität der $\gamma$-Effekte nachzuweisen.

$\gamma$-Aktivität ist jedoch aus einem weiteren, nicht direkt mit der Wahrnehmung zusammenhängenden Grund für den 


\section{Zusammenfassung $\cdot$ Abstract}

Tinnitus wichtig: Das relevante Zeitfenster zur Induktion synaptischer Plastizität liegt im Bereich eines $\gamma$-Zyklus ( $<40 \mathrm{~ms}$; [15]). Man kann somil davon ausgehen, dass fortdauernde $\gamma$-Aktivität zu einer Reorganisation der betroffenen Areale führt. Eine Reorganisation sensorischer Karten wurde bei Tinnitus sowie bei Phantomschmerz belegt $[16,17]$. Obwohl Kartenreorganisation nicht als neuronales Korrelat der Wahrnehmung im engeren Sinne interpretierbar ist, repräsentiert sie evtl, über $\gamma$-Oszillationen formierte stabile und "sparsame" ("sparse") Netze, in denen sich Erregung schnell und effizient ausbreitet. Ein solches Netz könnte in einem chronischen Zustand schwer aufzulösen sein und zur notorischen Therapieresistenz von Tinnitus beitragen.

$\mathrm{Zu}$ dieser Überlegung ist eine aktuelle MEG-Studie unserer Gruppe relevant [32], in der wir die unmittelbare Auswirkung eines Lärmtraumas auf die Spontanaktivität im auditorischen Kortex untersuchten. Wir wählten Hobby-Rockmusiker als Probanden aus, die über regelmäBigen Tinnitus nach Bandproben berichteten. Bei 13 von 14 Probanden beobachteten wir deutliche Erhöhungen der $\gamma$-Aktivität nach den Bandproben im rechten auditorischen Kortex (ם Abb. 2). Dieser stark lateralisierte Effekt korrespondierte nicht mit der Wahrnehmung, sondern mit einer deutlich stärker ausgeprägten Diskontinuität in der Hörfähigkeit für das linke Ohr im Vergleich zum rechten. Auf der Grundlage dieser Befunde nehmen wir an, dass ein Lärmtrauma unmittelbar zu einer Disinhibierung im auditorischen Kortext führt und unmittelbar Prozesse synaptischer Plastizität einsetzen. Es ist zu vermuten, dass dieser Zustand - falls fortdauernd - zu stabilen funktionellen und strukturellen Veränderungen im auditorischen Kortex führt.

Diese Untersuchung deutet auf ein anderes Muster der Spontanaktivitätsveränderung bei transientem als bei chronischem Tinnitus hin: Während im MEG die dominante Veränderung bei transientem Tinnitus die $\gamma$-Erhöhung ist, fällt bei chronischem Tinnitus v. a. die a-Reduktion auf. Dies legt nahe, dass tinnitusrelevante neuronale Prozesse sich evtl. im Zeitverlauf verändern. Da aktuelle tierexperimentelle Ergebnisse nahezu aus-

\section{N. Weisz $\cdot$ B. Langguth \\ Kortikale Plastizität und Veränderungen bei Tinnitus. Therapeutische Optionen}

\section{Zusammenfassung}

Ein wachsender Konsens in der aktuellen Tinnitusforschung legt zentralnervöse Veränderungen als Ursache für Tinnitus nahe. Zahlreiche tier- und humanexperimentelle Arbeiten konnten veränderte tonotope Repräsentationen sowie Spontanaktivität im auditorischen Kortex zeigen. Ein kausaler Zusammenhang zwischen veränderten neurophysiologischen Prozessen und Aspekten des Tinnitus wurde aber noch nicht nachgewiesen. Des Weiteren ist es wahrscheinlich, dass die Relevanz verschiedener Prozesse sich mit fortschreitender Tinnitusdauer ändert. Diese offenen Fragen erschweren die Entwicklung

wirksamer Behandlungen. Dennoch stehen heute zahlreiche neurowissenschaftlich motivierte Behandlungen zur Verfügung bzw. lassen sich in ein neurowissenschaftliches Rahmenmodell integrieren. Dieser Artikel gibt einen Überblick über aktuelle neurowissenschaftliche Entwicklungen in der Tinnitusforschung und diskutiert ihre Implikationen für die Behandlung von Tinnitus.

\section{Schlüsselwörter}

Plastizität, neuronale - Biofeedback - Tinnitus . Elektroenzephalographie - Magnetoenzephalographie

\section{Cortical plasticity and changes in tinnitus. Treatment options}

Abstract

A growing consensus in current tinnitus research suggests central nervous changes as the cause of tinnitus. Several animal and human experimental studies were able to show altered tonotopic representations as well as spontaneous activity in the auditory cortex. However, a causal relationship between altered neurophysiological processes and aspects of tinnitus are still missing. Furthermore, it is likely that the importance of diverse processes changes with continuing duration of tinnitus. These open questions complicate the development of effective treat- ments. Nevertheless, today several neuroscientifically motivated treatments are available, or treatments that can be integrated into a neuroscientific framework. This article gives an overview of current neuroscientific developments in tinnitus research and discusses their implications for the treatment of tinnitus.

\section{Keywords}

Plasticity, neuronal - Biofeedback - Tinnitus . Electroencephalography - Magnetoencephalography 


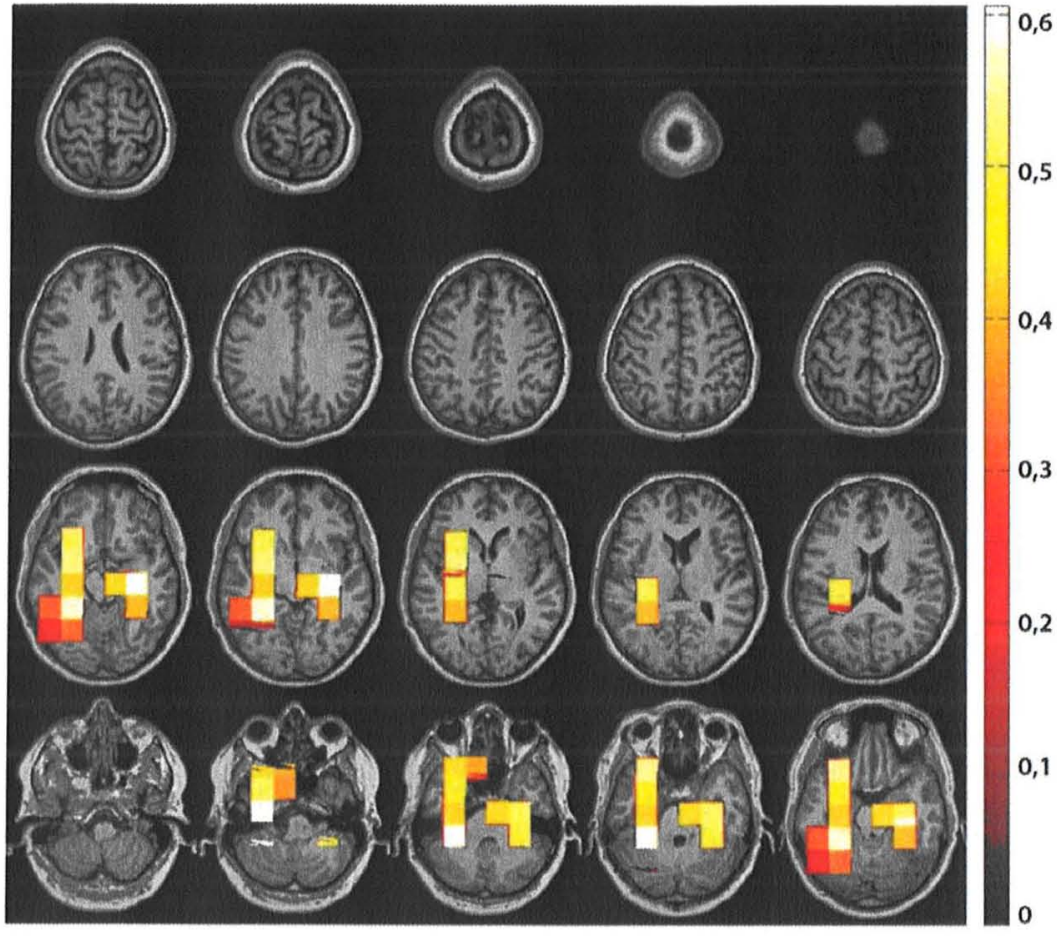

Abb. 3 A Je stärker die Tinnitusbelastung, desto mehr werden bilaterale temporale Areale von anderen Regionen beeinflusst

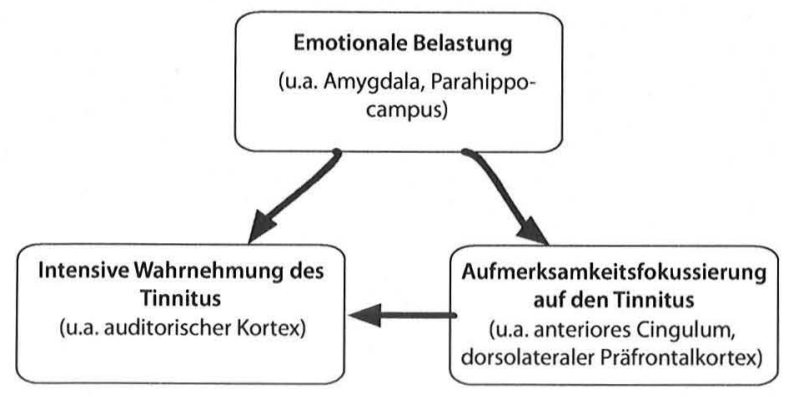

Abb. $4<$ Circulus vitiosus der Tinnituswahrnehmung

schließlich an Modellen des akuten Tinnitus gewonnen worden sind, sind sie nur bedingt auf chronischen Tinnitus übertragbar.

\section{Tinnitus und verteilte Netze}

Um Tinnitus in seiner Komplexität zu verstehen, reicht es nicht aus, Aktivitätsveränderungen im auditorischen Kortex $\mathrm{zu}$ beschreiben. Erst wenn eine Verbindung zu höhergeschalteten Gehirnarealen, quasi dem „Bewusstseinsnetz" erfolgt, wird aus der sensorischen Aktivität eine bewusste Wahrnehmung [18]. Darüber hinaus sind am interindividuell stark variierenden psychischen Leidensdruck emotions- und aufmerksamkeitsrelevante
(>4 Jahre) deutlich verteilter. Dieser Befund hat eine wichtige klinische Implikation für zielgerichtete Therapiemaßnahmen. Während eine Modulation der Aktivität im linken temporalen Kortex bei kurzer Tinnitusdauer hocheffektiv sein kann, da dadurch ein wichtiger Knotenpunkt im pathologischen Netz verändert wird, wird die gleiche Maßnahme bei längerer Tinnitusdauer geringere Effekte zeigen. Dies könnte die Abnahme der Wirksamkeit der transkraniellen Magnetstimulation mit fortschreitender Tinnitusdauer erklären.

Unsere Arbeitsgruppe interessiert sich zunehmend für die Beschreibung abstrakter Netzmaße bei Tinnitus. In einem ersten Ansatz untersuchten wir die Frage nach relevanten Knotenpunkten in einem funktionellen Tinnitusnetz, d. h. in Hirnregionen, die ein überproportionales Ausmaß an funktionellen Verknüpfungen mit anderen Hirnregionen unterhalten (sog. Hubs; [21]). Außerdem interessierten wir uns dafür, in welcher Richtung diese Beeinflussung erfolgt. Wir haben gezeigt, dass bei Tinnitusbetroffenen v. a. frontale und parietale Areale Hubs darstellen, die andere Hirnregionen in ihrer Aktivität beeinflussen. Dabei zeigte sich, dass der Einfluss auf temporale Areale stark in Abhängigkeit vom psychischen Leidensdruck moduliert wird (- Abb. 3). Dies belegt, dass die auditorische Aktivität bei Tinnitusbetroffenen durch kognitive und emotionale Mechanismen beeinflusst wird. Weitere Konnektivitätsstudien werden uns dem Verständnis sowie der Behandlung von Tinnitus einen Schritt näherbringen.

\section{Neurowissenschaftlich basierte Tinnitusbehandlungen}

Mit Tinnitus assoziierte neuronale Netze umfassen sowohl Veränderungen im auditorischen Kortex als auch in „höheren“ frontalen und parietalen Gehirnarealen. Alle beteiligten Gehirnareale stellen mögliche Ansatzpunkte für die Behandlung von Tinnitus dar. Neurowissenschaftlich fundierte Behandlungen lassen sich grob danach unterteilen, ob sie versuchen,

- das der Wahrnehmung zugrunde liegende Signal im auditorischen Kortex direkt zu beeinflussen [1] oder 
- höhere Areale, die für die Wahrnehmung, Aufmerksamkeitsfokussierung und affektive Bewertung relevant sind [2], oder

- die Kopplung zwischen diesen Arealen [3].

Eine Unterteilung in kausale und nichtkausale Therapien ist in Anbetracht einer "Netzperspektive“ überholt.

\section{Psychotherapie und Neurobiofeedback}

Die Psychotherapie gehört zu den etabliertesten Behandlungsmethoden des chronischen Tinnitus [22]. Die Zielsetzung der verschiedenen psychotherapeutischen Methoden besteht darin, über eine kognitive Umstrukturierung und eine Veränderung des Verhaltens einen weniger belastenden Umgang mit dem Tinnitus zu erlernen. Insbesondere geht es darum, den Circulus vitiosus, der dem schweren Tinnitus häufig zugrunde liegt, zu durchbrechen (- Abb.4).

\section{(2) Die Zielsetzung der Psychotherapie ist ein weniger belastender Umgang mit dem Tinnitus}

Auch wenn bisher keine Untersuchungen vorliegen, die den Einfluss einer erfolgreichen Psychotherapie auf das neuronale Aktivitätsmuster bei Tinnitus untersucht haben, ist anzunehmen, dass die subjektive Besserung mit einer Verminderung der gesteigerten neuronalen Aktivität in den entsprechenden Gehirnarealen und einer Normalisierung der gesteigerten Verbindung zwischen den Gehirnarealen einhergeht. Hinweise dafür, welchen Einfluss Verhaltensmaßnahmen auf die $\mathrm{Ge}$ hirnaktivität von Tinnituspatienten haben können, finden sich in einer Studie, die die Aktivierung des auditorischen Kortex bei Tinnituspatienten mit Hilfe der Positronenemissionstomographie untersucht hat: Das Lösen einer einfachen mathematischen Aufgabe führte zur verminderten Tinnituswahrnehmung und gleichzeitig auch zur Verminderung der neuronalen Aktivität im auditorischen Kortex [23]. Gehirnaktivitätsmessungen vor und nach erfolgreichen psychotherapeutischen In-

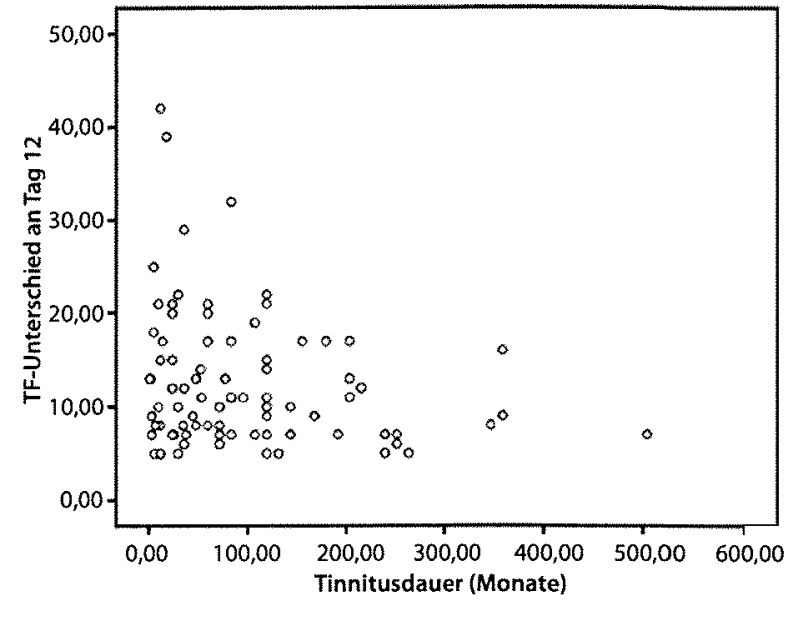

terventionen würden wesentlich dazu beitragen, die exakten Elemente der Therapie $z u$ identifizieren und diese dann systematisch zu verbessern.

Ähnlich wie bei der Psychotherapie werden beim Neurobiofeedback Topdown-Mechanismen ausgenutzt. Dabei werden operante Lernmechanismen eingesetzt, d. h. der Betroffene lernt durch eine Rückmeldung, ein für den Tinnitus relevantes Aktivitätsmerkmal willentlich zu beeinflussen. Häufig werden dabei bestimmte EEG-Spektren als Signal verwendet. In einer EEG-Studie haben wir gezeigt [24], dass eine durch Neurobiofeedback erzielte Normalisierung des $a-\delta$ Quotienten mit einer Reduktion der Tinnitusintensität einhergeht. Allerdings ist aus dieser Studie nicht klar ersichtlich, ob diese Veränderung v. a. der Aktivität im auditorischen Kortex zuzuschreiben ist. Um dieses Ziel zu erreichen, muss sich jedoch insgesamt die methodische Qualität von Neurofeedbackansätzen erhöhen, u. a.

möglichst präzises Feedback aus enger umschriebenen Hirnregionen,

d. h. Einsatz einer ausreichenden Zahl von EEG-Elektroden ( $>60$ ), kombiniert mit modernen Quellenlokalisationsverfahren,

gute Kontrolle externer und körperlicher Artefaktquellen (z. B. Augenbewegungen, Muskelaktivität).

Des Weiteren ist davon auszugehen, dass Neurofeedback umso effizienter ist, je genauer die dem Tinnitus zugrunde liegenden neuronalen Veränderungen im indi- viduellen Fall identifiziert werden können.

\section{Akustische und Hirnstimulation}

Neben den oben beschriebenen Ansätzen zur therapeutischen Beeinflussung der Top-down-Modulationen mehren sich therapeutische Versuche, abnormale Aktivität im auditorischen Kortex direkt zu normalisieren. Eine Strategie dabei ist es, den fehlenden akustischen Input via passive akustische Stimulation, z. B. durch Hörgeräte oder Cochlear implants, zu kompensieren und somit den maladaptiven neuroplastischen Prozessen die Grundlage zu entziehen. Über positive Effekte auf Tinnitus durch Cochlear implants bei einseitiger Taubheit wurde wiederholt berichtet [25]. Auch Berichte aus der Praxis, dass das Tragen von Hörgeräten die Intensität des Tinnitus reduziert, deuten an, dass eine systematisch eingesetzte akustische Stimulation therapeutisch wirksam sein kann.

Tierexperimentell wurde nachgewiesen, dass eine massive akustische Stimulation im Hochtonbereich unmittelbar nach einem Lärmtrauma sowohl das Ausmaß des Hörverlusts als auch die Reorganisation der tonotopen Karte im primär auditorischen Kortex reduziert [26]. Die Übertragung auf den Humanbereich wird allerdings dadurch erschwert, dass die akustische Stimulation unmittelbar nach dem Lärmtrauma erfolgte und dass es unklar ist, ob die Tiere Tinnitus wahrnahmen, und wenn ja, ob der Tinnitus für die Tiere belastend war. Erste Versuche, diesen An- 
satz auf chronischen Tinnitus beim Menschen anzuwenden, ergaben keine nachweisbare Besserung des Tinnitus [27]. AIlerdings reduzierte sich die Hyperakusis, wobei es sich wahrscheinlich um eine Form der Desensibilisierung handelt.

Neben der Tatsache, dass ein stabilisiertes tinnitusrelevantes neuronales Netz (d. h. in einem chronischen Zustand) möglicherweise nur schwer zu beeinflussen ist, ist ein Problem akustischer Ansätze, dass Schädigungen der Cochlea schwer vorherzusagende Veränderungen der Frequenzwahrnehmung mit sich bringen. Ein Beispiel dafür ist „off-frequency listening “ [2], d. h. dass ein akustischer Reiz mit einer Frequenz in einem stark geschädigten Bereich der Cochlea mit relativ gesunden benachbarten Haarzellen verarbeitet wird (ermöglicht durch die breite Ausdehnung der Wanderwelle). Praktisch ist es aus einem Audiogramm kaum möglich vorherzusagen, welche Regionen im auditorischen Kortex ein definierter akustischer Reiz aktiviert, sodass es leicht passieren kann, dass andere als die intendierten Regionen stimuliert werden. Diese Tatsache könnte auch für den relativ geringen Erfolg bzw. die inkonsistenten Resultate von Frequenzdiskriminationstraining $[28,29]$ verantwortlich sein, ein Ansatz, von dem man sich nach dem Beleg verzerrter tonotoper Karten beim Menschen [17] viel erhoffte.

Dass gezielte akustische Stimulation durchaus wirksam sein kann, belegt eine neue Studie [30]. In diesem Ansatz nutzten die Forscher Musik, aus der bestimmte Frequenzbereiche um die Tinnitusfrequenz herum herausgefiltert wurden. Gegenüber einer Placebobedingung reduzierte sich die subjektive Tinnituslautheit deutlich, jedoch ist die absolute Reduktion von etwa $20-30 \%$ über ein Jahr durchaus vergleichbar mit anderen Therapien, z. B. Neurofeedback. Die besondere Bedeutung dieser Studie liegt im Beleg, dass eine Verminderung der Tinnitussymptomatik einherging mit einer Verminderung eines neuronalen Markers - und zwar mit einer reduzierten evozierten Gehirnaktivität auf akustische Reize in der Tinnitusfrequenz. Der Grund, warum diese Studie im Vergleich zu den oben erwähnten akustischen Stimulationsansätzen erfolgreicher war, liegt höchstwahrscheinlich in einer sorgfältigen Selektion der Probanden: Diese durften zum einen kaum Hörverlust aufweisen und mussten zum anderen in der Lage sein, ihre Tinnitusfrequenz zuverlässig einschätzen zu können. Einschränkend ist auch zu erwähnen, dass die Wirksamkeit dieses vielversprechend erscheinenden Therapieansatzes erst in weiteren Studien auch bei Patienten mit Hörverlust bestätigt werden muss, bevor ein Einsatz in der Routinebehandlung empfohlen werden kann.

Ein alternativer Ansatz ist die direkte Stimulation relevanter auditorischer kortikaler Regionen, z. B. durch repetitive transkranielle Magnetstimulation (rTMS) oder implantierte Elektroden. Im motorischen System haben bestimmte Stimulationsformen (z. B. 1- oder 10-Hz-rTMS) Veränderungen kortikaler Erregung gezeigt, die die Dauer der Stimulation überdauerten. Ließe sich dieses Prinzip auf das auditorische System übertragen, dann wäre rTMS konzeptuell ein wirkungsvoller Ansatz, um die angenommene Übererregung im auditorischen Kortex bei Tinnitusbetroffenen positiv therapeutisch zu beeinflussen. Zahlreiche placebokontrollierte Studien mittels $1-\mathrm{Hz}$-rTMS haben statistisch signifikante Reduktionen von Tinnitus ergeben. Allerdings ist der Effekt mit im Durchschnitt maximal 20-30\% Symptomreduktion relativ gering und die interindividuelle Variabilität enorm hoch. Übereinstimmend findet sich in verschiedenen Studien ein wesentlicher Einfluss der Tinnitusdauer auf das Behandlungsergebnis ([31]; - Abb. 5).

Dieser Befund unterstreicht die Annahme, dass bei Tinnitus im Zeitverlauf Veränderungen der zugrunde liegenden neuronalen Mechanismen stattfinden, die unterschiedliche therapeutische Strategien für verschiedene Chronifizierungsgrade erfordern. Auch sind sowohl weitere grundlagenwissenschaftliche als auch $\mathrm{kli}$ nische Untersuchungen notwendig, bevor eine Einschätzung getroffen werden kann, ob rTMS eine Option für die Routinebehandlung von Tinnitus darstellt. Wesentliche zu klärende Fragen umfassen die Frage der optimalen Lokalisation und des optimalen Behandlungsprotokolls für den einzelnen Patienten. Wir sind zuversichtlich, dass eine Beantwortung dieser Fragen die Wirksamkeit von rTMS in Zu- kunft verbessern wird. Darüber hinaus sind - basierend auf einem detaillierten Verständnis der neurobiologischen Effekte der TMS - auch Kombinationsbehandlungen mit auditorischer oder pharmakologischer Stimulation oder der kombinierte synergistische Einsatz mit Neurobiofeedback oder verhaltenstherapeutischen Ansätzen denkbar.

\section{Ausblick}

Obwohl in den letzten 20 Jahren große Fortschritte im neurowissenschaftlichen Verständnis von Tinnitus zu verzeichnen sind, haben diese Bemühungen noch nicht zu einem Durchbruch in der Therapie des Tinnitus geführt - in dem Sinne, dass der Tinnitus reliabel reduziert oder gar eliminiert werden könnte. Ebenso ist ein objektiver diagnostischer Marker, der im Einzelfall diagnostisch aussagekräftig ist, trot $z$ aller Bemühungen noch nicht in Sicht. Gründe dafür sind im Wesentlichen die immer noch geringe Zahl von Studien zu den grundlegenden neuronalen Mechanismen der verschiedenen Tinnitusformen.

Dank erheblicher Fortschritte in den letzten Jahren stehen jedoch sowohl im Bereich tierexperimenteller Untersuchungen als auch im Bereich der klinischen Forschung Methoden zur Verfügung, mit denen wesentliche offene Fragen experimentell angegangen werden können, u. a.

Was ist das grundlegende neuronale Korrelat der Tinnituswahrnehmung?

- Wie ist das verteilte funktionelle Netz konstituiert, das die bewusste Wahrnehmung sowie affektive Aspekte repräsentiert?

- Wie findet die Kommunikation innerhalb eines solchen Netzes statt?

Auch die Identifikation protektiver Faktoren ist sowohl von grundlagenwissenschaftlicher als auch von klinischer Bedeutung, u. a. zur Beantwortung folgender Fragen:

Warum entwickeln nicht alle Personen mit Hörverlust Tinnitus?

Warum entwickeln manche Betroffene chronischen Tinnitus, während bei anderen die Wahrnehmung nach einiger Zeit verschwindet? 


\section{Fazit für die Praxis}

Trotz der ungeklärten Fragen lassen sich bestimmte Aussagen für die Praxis treffen:

Verschiedene therapeutische Ansätze wirken neuronal gesehen auf verschiedenen Ebenen. Es ist wesentlich, dass Kliniker ihre Therapieansätze bzgl. des Wirkorts im neuronalen Tinnitusnetz einzuordnen wissen.

Es existieren verschiedene Formen von Tinnitus. Klinische Kriterien, wie z. B. die Beschaffenheit des Hörverlusts, die Tinnitusdauer oder die psychische Belastung, spiegeln sich in spezifischen neuronalen Korrelaten wider.

Die Auswahl des optimalen therapeutischen Ansatzes wird entscheidend von der genauen klinischen und möglicherweise auch neurobiologischen Charakterisierung des einzelnen Tinnituspatienten abhängen. Eventuell ist in manchen Fällen eine Kombination verschiedener Ansätze wirksamer als ein einzelnes Verfahren.

- Psychotherapeutische und Neurofeedbackansätze nutzen Top-downModulationen aus und sind somit wahrscheinlich relativ universell einsetzbar.

Akustische Stimulationen sind nicht sinnvoll, wenn der Tinnitus in Bereiche mit starkem Hörverlust fällt, v. a. bei geschädigten inneren Haarzellen (s. "off-frequency listening"). Dabei ist zur Abschätzung des Hörverlusts ein Routineaudiogramm nicht ausreichend.

Bei moderatem Hörverlust und klaren Vorstellungen, welche Frequenzbereiche relevant sind, können Verfahren mit akustischer Stimulation helfen, Tinnitus zu reduzieren. Des Weiteren deuten tierexperimentelle Studien an, dass akustische Stimulation unmittelbar nach dem Tinnitus auslösenden Ereignis sowohl Hörverlust als auch maladaptive neuroplastische Prozesse vermindern kann. Daraus lässt sich die Hypothese ableiten, dass systematisch eingesetzte akustische Stimulation in einer sehr frühen Phase nach Tinnitusbeginn einer Chronifizierung vorbeugen könnte.
Direkte Hirnstimulationen des akustischen Kortex, z. B. mittels rTMS, sind relativ ungeeignet zur Behandlung von sehr lang andauerndem Tinnitus.

\section{Korrespondenzadresse}

N.Weisz

Fachbereich Psychologie

Universität Konstanz

D23

78457 Konstanz

nathan.weisz@uni-konstanz.de

Interessenkonflikt. Der korrespondierende Autor gibt an, dass kein interessenkonflikt besteht.

\section{Literatur}

1. Eggermont JJ, Roberts LE (2004). The neuroscience of tinnitus. Trends Neurosci 27:676-682

2. Weisz N, Hartmann T, Dohrmann K et al (2006) High-frequency tinnitus without hearing loss does not mean absence of deafferentation. Hear Res 222:108-114

3. Konig O, Schaette R, Kempter R, Gross M (2006) Course of hearing loss and occurrence of tinnitus. Hear Res 221:59-64

4. Norena AJ, Eggermont JJ (2003) Changes in spontaneous neural activity immediately after an acoustic trauma: implications for neural correlates of tinnitus. Hear Res 183:137-153

5. Weisz N, Dohrmann K Elbert T (2007) The relevance of spontaneous activity for the coding of the tinnitus sensation. Prog Brain Res 166:61-70

6. Moazami-Goudarzi M, Michels L, Weisz N, Jeanmonod $D(2010)$ Temporo-insular enhancement of EEG low and high frequencies in patients with chronic tinnitus. QEEG study of chronic tinnitus patients. BMC Neurosci 11:40

7. Loo E van der, Gais S, Congedo M et al (2009) Tinnitus intensity dependent gamma oscillations of the contralateral auditory cortex. PLoS One 4:e7396

8. Llinas RR, Ribary U, Jeanmonod D et al (1999) Tinnitus intensity dependent gamma oscillations of the contralateral auditory cortex. Proc Natl Acad SciU S A 96:15222-15227

9. Romei V, BrodbeckV, Michel C et al (2008) Spontaneous fluctuations in posterior alpha-band EEG activity reflect variability in excitability of human visual areas. Cereb Cortex 18:2010-2018

10. Sauseng P, Klimesch W, Gerloff C, Hummel FC (2009) Spontaneous locally restricted EEG alpha activity determines cortical excitability in the motor cortex. Neuropsychologia 47:284-288

11. Fries $P$, Nikolic $D$, Singer $W(2007)$ The gamma cycle. Trends Neurosci 30:309-316

12. Bertrand O, Tallon-Baudry C (2000) Oscillatory gamma activity in humans: a possible role for object representation. Int J Psychophysiol 38:211 223

13. Wyart V, Tallon-Baudry C (2009) How ongoing fluctuations in human visual cortex predict perceptual awareness: baseline shift versus decision bias. Neurosci 29:8715-8725

14. Weisz N, Muller S, Schlee W et al (2007) The neural code of auditory phantom perception. I Neurosci 27:1479-1484
5. Bi G, Poo M (2001) Synaptic modification by correlated activity: Hebb's postulate revisited. Annu Rev Neurosci 24:139-166

16. Flor H, Elbert T, Knecht $\mathrm{S}$ et al (1995) Phantomlimb pain as a perceptual correlate of cortical re organization following arm amputation. Nature 375:482-484

17. Muhlnickel W, Elbert T, Taub E, Flor H (1998) Reorganization of auditory cortex in tinnitus. Proc Nat Acad Sci U S A 95:10340-10343

18. Dehaene $S$, Changeux JP, Naccache L et al (2006) Conscious, preconscious, and subliminal processing: a testable taxonomy. Trends Cogn Sci 10:204-211

19. Schlee W, Weisz N, Bertrand O et al (2008) Using auditory steady state responses to outline the functional connectivity in the tinnitus brain. PLos One $3: 3720$

20. Schlee W, Hartmann T, Langguth B, Weisz N (2009) Using auditory steady state responses to outline the functional connectivity in the tinnitus brain. BMC Neurosci 10:11

21. Schlee W, Mueller N, Hartmann T et al (2009) Mapping cortical hubs in tinnitus. BMC Biol 7:80

22. Phillips JS, McFerran D (2010) Tinnitus retraining therapy (TRT) for tinnitus. Cochrane Database Syst Rev 3, CD007330

23. Andersson $G$, Juris $L$, Classon E et al (2006) Consequences of suppressing thoughts about tinnitus and the effects of cognitive distraction on brain activity in tinnitus patients. Audiol Neurootol 11:301-309

24. Dohrmann K, Weisz N, Schlee W et al (2007) Neurofeedback for treating tinnitus. Prog Brain Res 166:473-485

25. Van de Heyning P, Vermeire $K$, Diebl M et al (2008) Incapacitating unilateral tinnitus in single-sided deafness treated by cochlear implantation. Ann Otol Rhinol Laryngol 117:645-652

26. Norena AJ, Eggermont J) (2005) Enriched acoustic environment after noise trauma reduces hearing loss and prevents cortical map reorganization. J Neurosci 25:699-705

27. Norena AJ, Chery-Croze S (2007) Enriched acoustic environment rescales auditory sensitivity. Neuroreport 18:1251-1255

28. Flor H, Hoffmann D, Struve M, Diesch E (2004) Auditory discrimination training for the treatment of tinnitus. Appl Psychophysiol Biofeedback 29:113120

29. Herraiz $C$, Diges $I$, Cobo P et al (2010) Auditory discrimination training for tinnitus treatment: the effect of different paradigms. Eur Arch Otorhinolaryngol 267(7):1067-1074

30. Okamoto H, Stracke H, Stoll W, Pantev C (2010) Listening to tailor-made notched music reduces tinnitus loudness and tinnitus-related auditory cortex activity. Proc Natl Acad Sci U S A 107:1207-1210

31. Frank $G$, Kleinjung T, Landgrebe $M$ et al (2010) Listening to tailor-made notched music reduces tinnitus loudness and tinnitus-related auditory cortex activity. Eur J Neurol 17(7):951-956

32. Ortmann M, Müller N, Schlee W, Weisz N (2010) Rapid increases of gamma power in the auditory cortex following noise trauma in humans. Eur I Neurosci; (eingereicht) 